\title{
Propensity score analysis of radical cystectomy versus bladder-sparing trimodal therapy in the setting of a multidisciplinary bladder cancer clinic
}

Kulkarni, Girish S ; Hermanns, Thomas ; Wei, Yanliang ; Bhindi, Bimal ; Satkunasivam, Raj ; Athanasopoulos, Paul ; Bostrom, Peter J ; Kuk, Cynthia ; Li, Kathy ; Templeton, Arnoud J ; Sridhar, Srikala S ; van der Kwast, Theodorus H ; Chung, Peter ; Bristow, Robert G ; Milosevic, Michael ; Warde, Padraig ; Fleshner, Neil E ; Jewett, Michael A S ; Bashir, Shaheena ; Zlotta, Alexandre R

\begin{abstract}
Purpose Multidisciplinary management improves complex treatment decision making in cancer care, but its impact for bladder cancer (BC) has not been documented. Although radical cystectomy (RC) currently is viewed as the standard of care for muscle-invasive bladder cancer (MIBC), radiotherapybased, bladder-sparing trimodal therapy (TMT) that combines transurethral resection of bladder tumor, chemotherapy for radiation sensitization, and external beam radiotherapy has emerged as a valid treatment option. In the absence of randomized studies, this study compared the oncologic outcomes between patients treated with RC or TMT by using a propensity score matched-cohort analysis. Methods Data from patients treated in a multidisciplinary bladder cancer clinic (MDBCC) from 2008 to 2013 were reviewed retrospectively. Those who received TMT for MIBC were identified and matched (for sex, cT and $\mathrm{cN}$ stage, Eastern Cooperative Oncology Group status, Charlson comorbidity score, treatment date, age, carcinoma in situ status, and hydronephrosis) with propensity scores to patients who underwent RC. Overall survival and disease-specific survival (DSS) were assessed with Cox proportional hazards modeling and a competing risk analysis, respectively. Results A total of 112 patients with MIBC were included after matching ( 56 who had been treated with TMT, and 56 who underwent RC). The median age was 68.0 years, and $29.5 \%$ had stage cT3/cT4 disease. At a median follow-up of 4.51 years, there were 20 deaths $(35.7 \%)$ in the RC group (13 as a result of BC) and 22 deaths $(39.3 \%)$ in the TMT group (13 as a result of BC). The 5 -year DSS rate was $73.2 \%$ and $76.6 \%$ in the RC and TMT groups, respectively ( $\mathrm{P}=.49)$. Salvage cystectomy was performed in $6(10.7 \%)$ of 56 patients who received TMT. Conclusion In the setting of a MDBCC, TMT yielded survival outcomes similar to those of matched patients who underwent RC. Appropriately selected patients with MIBC should be offered the opportunity to discuss various treatment options, including organ-sparing TMT.
\end{abstract}

DOI: https://doi.org/10.1200/JCO.2016.69.2327

Posted at the Zurich Open Repository and Archive, University of Zurich

ZORA URL: https://doi.org/10.5167/uzh-142705

Journal Article

Published Version

Originally published at:

Kulkarni, Girish S; Hermanns, Thomas; Wei, Yanliang; Bhindi, Bimal; Satkunasivam, Raj; Athanasopoulos, Paul; Bostrom, Peter J; Kuk, Cynthia; Li, Kathy; Templeton, Arnoud J; Sridhar, Srikala S; van der Kwast, Theodorus H; Chung, Peter; Bristow, Robert G; Milosevic, Michael; Warde, Padraig; Fleshner, 
Neil E; Jewett, Michael A S; Bashir, Shaheena; Zlotta, Alexandre R (2017). Propensity score analysis of radical cystectomy versus bladder-sparing trimodal therapy in the setting of a multidisciplinary bladder cancer clinic. Journal of Clinical Oncology, 35(20):2299-2305.

DOI: https://doi.org/10.1200/JCO.2016.69.2327 
Author affiliations and support information (if applicable) appear at the end of this article.

Published at jco.org on April 14, 2017 G.S.K. and T.H. contributed equally to this work.

Corresponding author: Alexandre R. Zlotta, MD, PhD, Department of Surgery, Division of Urology, Mount Sinai Hospital, 60 Murray St, 6th floor, Room 6004, Box 19, Toronto, Ontario M5T 3L9, Canada; e-mail: alexandre.zlotta@

sinaihealthsystem.ca.

() 2017 by American Society of Clinical Oncology

0732-183X/17/3520w-2299w/\$20.00

\section{Propensity Score Analysis of Radical Cystectomy Versus Bladder-Sparing Trimodal Therapy in the Setting of a Multidisciplinary Bladder Cancer Clinic}

Girish S. Kulkarni, Thomas Hermanns, Yanliang Wei, Bimal Bhindi, Raj Satkunasivam, Paul Athanasopoulos, Peter J. Bostrom, Cynthia Kuk, Kathy Li, Arnoud J. Templeton, Srikala S. Sridhar, Theodorus H. van der Kwast, Peter Chung, Robert G. Bristow, Michael Milosevic, Padraig Warde, Neil E. Fleshner, Michael A.S. Jewett, Shaheena Bashir, and Alexandre R. Zlotta

\section{$\begin{array}{llllllll}\text { A } & \text { B } & \mathbf{S} & \mathbf{T} & \mathbf{R} & \mathbf{A} & \mathbf{C} & \mathbf{T}\end{array}$}

\section{Purpose}

Multidisciplinary management improves complex treatment decision making in cancer care, but its impact for bladder cancer (BC) has not been documented. Although radical cystectomy (RC) currently is viewed as the standard of care for muscle-invasive bladder cancer (MIBC), radiotherapybased, bladder-sparing trimodal therapy (TMT) that combines transurethral resection of bladder tumor, chemotherapy for radiation sensitization, and external beam radiotherapy has emerged as a valid treatment option. In the absence of randomized studies, this study compared the oncologic outcomes between patients treated with RC or TMT by using a propensity score matched-cohort analysis.

\section{Methods}

Data from patients treated in a multidisciplinary bladder cancer clinic (MDBCC) from 2008 to 2013 were reviewed retrospectively. Those who received TMT for MIBC were identified and matched (for sex, cT and cN stage, Eastern Cooperative Oncology Group status, Charlson comorbidity score, treatment date, age, carcinoma in situ status, and hydronephrosis) with propensity scores to patients who underwent RC. Overall survival and disease-specific survival (DSS) were assessed with Cox proportional hazards modeling and a competing risk analysis, respectively.

\section{Results}

A total of 112 patients with MIBC were included after matching (56 who had been treated with TMT, and 56 who underwent RC). The median age was 68.0 years, and $29.5 \%$ had stage cT3/cT4 disease. At a median follow-up of 4.51 years, there were 20 deaths $(35.7 \%$ ) in the RC group (13 as a result of BC) and 22 deaths (39.3\%) in the TMT group (13 as a result of BC). The 5-year DSS rate was $73.2 \%$ and $76.6 \%$ in the RC and TMT groups, respectively $(P=.49)$. Salvage cystectomy was performed in $6(10.7 \%)$ of 56 patients who received TMT.

\section{Conclusion}

In the setting of a MDBCC, TMT yielded survival outcomes similar to those of matched patients who underwent RC. Appropriately selected patients with MIBC should be offered the opportunity to discuss various treatment options, including organ-sparing TMT.

\section{J Clin Oncol 35:2299-2305. (C) 2017 by American Society of Clinical Oncology}

\section{INTRODUCTION}

Bladder cancer (BC) care can be complex. Variation in assessment of pathologic stage, grade, and histologic subtype, quality of care, ${ }^{1,2}$ or utilization of perioperative chemotherapy ${ }^{3,4}$ affect management and outcomes. ${ }^{5}$ Multidisciplinary care (MDC) models have been shown to maximize outcomes by minimizing such variability, which reduces judgment errors and optimizes communication among physicians and between caregivers and patients. ${ }^{6-9}$ MDC provides an opportunity for all treatment options to be discussed, which is important for BC care, in which multiple treatment options are available to patients regardless of the stage at presentation.

The traditional treatment of muscle-invasive bladder cancer (MIBC) has been radical cystectomy (RC). Bladder-sparing strategies have emerged as 
a valid treatment option during the past decades. Recent data from many noncomparative single-arm series and Radiation Therapy Oncology Group protocols demonstrated that selected patients with MIBC experience excellent outcomes and experience preservation of a functioning bladder with trimodal therapy (TMT), which consists of debulking transurethral resection of bladder tumor (TURBT), chemotherapy for radiation sensitization, and external beam radiotherapy. ${ }^{10-13}$ To date, only one small, nonadjusted, case-control series of TMT versus RC in MIBC has been reported, which demonstrated no difference between groups. ${ }^{14}$ A propensity score adjusted direct comparison of TMT to RC in MIBC has not been reported.

Radiotherapy alone, radiotherapy and concurrent cisplatin chemotherapy, or neoadjuvant chemotherapy (NAC) followed by radiotherapy have been performed for more than three decades at the Princess Margaret Cancer Centre, University Health Network (UHN), Toronto, Canada. ${ }^{11}$ The increasing complexity of treatment decisions and the need to offer various treatment options, including RC and TMT, for eligible patients with MIBC were recognized, and a weekly multidisciplinary bladder cancer clinic (MDBCC) was established at the center in 2008. This study aimed to assess changes in care plans after multidisciplinary assessment and to compare oncologic outcomes between patients with MIBC who were treated with RC or TMT in the MDBCC by using a propensity score analysis.

\section{METHODS}

\section{MDBCC Overview}

The MDBCC was initiated as a weekly, referral-based outpatient clinic held in the cystoscopy suite of UHN, where patients with complex $\mathrm{BC}$ cases were referred for evaluation and second opinion by a multidisciplinary team that specialized in BC. Administrative coordinators prepared a summary of patient data, which included pathology review and imaging, before the patients' clinic visits. Clinical information was summarized in the bladder informatics tool (eCancerCareBladder), as reported previously. ${ }^{15}$

In the MDBCC, all patients were evaluated by a multidisciplinary team composed of one of four urologic oncologists (G.S.K., N.E.F., M.A.S.J., A.R.Z.) and one of four radiation oncologists (P.C., R.G.B., M.M., P.W.), all of whom have expertise in $\mathrm{BC}$ care. ${ }^{16} \mathrm{~A} \mathrm{BC}$ expert medical oncologist (S.S.S.) was available for immediate, same-day consultation. Pathology review by expert genitourinary (GU) pathologists was initiated in case of initial assessment by a non-GU pathologist, variant histology, or doubt of the TURBT stage. Patient assessment involved a thorough history and physical examination, detailed review of external reports and available imaging, and cystoscopic evaluation with surgical and radiation oncologists in attendance. After multidisciplinary discussion, consensus recommendations for additional investigations (eg, pathology review by uropathology, additional imaging, and/or transurethral reresections) or definitive treatment were made.

\section{MDBCC Patients}

All consecutive patients with $\mathrm{BC}$ referred to the MDBCC between April 2008 and December 2012 were identified retrospectively and included in the study to assess the impact of additional investigations and treatment decision in the clinic, and patients were identified and included up to December 2013 for the propensity score analysis. Baseline parameters of patients were recorded from electronic hospital charts. Electronically archived referral letters were used to identify the initial proposed treatment plan. Post-MDBCC disease stage and definitive treatment were recorded. Posttreatment outcome results were retrieved from electronic hospital charts. Institutional research ethics board approval was obtained (REB 10-0367-CE, 15-8727-CE).

\section{Treatment}

After clinic assessment and review of additional investigations and/or data (when necessary), final treatment decisions were presented to the patient with all physicians present. Patients with metastatic disease (regional and systemic) were referred for primary chemotherapy. Patients with localized non-muscle-invasive bladder cancer (NMIBC) were offered additional reresection, intravesical therapy, RC, radiotherapy, or enrollment in clinical trials. Patients with localized, nonmetastatic MIBC were assessed for their candidacy for TMT, RC, and/or clinical trials. RC consisted of cystoprostatectomy in men or anterior exenteration in women with bilateral pelvic lymph node dissection (template at the surgeon's discretion) and urinary diversion (ileal conduit, ileal neobladder, or Indiana pouch).

Patients with a history of pelvic radiotherapy who underwent RC were excluded from the propensity score analysis, because they would not be eligible for both treatment modalities. Candidates for TMT had (1) tumors $<5 \mathrm{~cm}$, (2) solitary tumors, (3) minimal to no hydronephrosis, (4) good bladder function, and (5) no multifocal carcinoma in situ (CIS). Patients with limited CIS adjacent to the primary tumor and mild hydronephrosis, and patients at high risk for RC morbidity and mortality were not excluded from TMT consideration. If patients were candidates for TMT and RC, both were discussed, and the discussion included expected outcomes and complications.

The merits of perioperative chemotherapy using a shared decisionmaking process (NAC for patients who received TMT, NAC $v$ adjuvant chemotherapy $[\mathrm{AC}]$ for patients who underwent $\mathrm{RC}$ ) were discussed with patients. The criteria for cisplatin-based NAC were an Eastern Cooperative Oncology Group (ECOG) status $<2$, creatinine clearance $>60 \mathrm{~mL} / \mathrm{min}$, no grade 2 or worse hearing loss or neuropathy, and adequate cardiac function (less than New York Heart Association class III heart failure). ${ }^{17}$ In addition to NAC criteria, AC was only offered to patients who underwent $\mathrm{RC}$ and who had pathologic stage pT3/4 and/or N+. NAC and AC consisted of four cycles of gemcitabine plus cisplatin. A debulking TURBT to remove all visible tumor was performed before concomitant chemoradiotherapy. Radio-opaque contrast (0.5-1 mL Lipiodol [ethiodized oil, Guerbet LLC, Bloomington, IN]) was injected cystoscopically at the periphery of the tumor resection site for radiation treatment planning and to facilitate radiation delivery. Daily image-guided intensity-modulated radiotherapy was delivered to the bladder and pelvic nodes to a dose of 46 Gy in 23 fractions with a sequential tumor boost of $20 \mathrm{~Gy}$ in 10 fractions (total, 66 Gy). Radiosensitization with concurrent single-agent cisplatin chemotherapy $\left(40 \mathrm{mg} / \mathrm{m}^{2}\right)$ was administered weekly during therapy.

\section{Follow-Up}

All patients were observed by a standardized protocol of clinic visits (quarterly visits in year 1, biannual visits in years 2 and 3, and annual visits thereafter). Visits involved history, physical examination, blood work, and urinary cytology. Patients who received TMT or RC underwent axial imaging (computed tomography or magnetic resonance imaging) biannually to detect recurrent disease and complications. Patients who received TMT also underwent cystoscopy and salvage RC if they developed MIBC. Random bladder biopsy was not routine. Intravesical therapy and the management of NMIBC intravesical recurrence were based on established guidelines. ${ }^{18-21}$

\section{Outcomes}

The immediate impact of MDBCC on BC management was assessed by determining how often MDBCC assessment changed the initial tumor stage and/or treatment plan. Any change in clinical tumor stage and/or 
treatment plan that resulted from MDBCC assessment was recorded. Change in treatment was attributed to revised stage and/or expert opinion. Overall survival (OS) and disease-specific survival (DSS) for patients who received TMT were compared with those propensity-matched patients who underwent RC.

\section{Statistical Analyses}

Short-term changes in stage and treatment plan were reported with descriptive statistics for continuous variables (means and medians) and categoric variables (percentages). To assess long-term outcomes, propensity score matching of patients treated with TMT to patients treated with RC at the UHN from 2008 to 2013 was performed. ${ }^{22}$ Only patients who had urothelial-predominant BC were included. Pretreatment clinical variables used for matching and for the propensity score analysis are detailed in the Appendix (online only). Patients were observed until death, loss to follow-up, or August 2016, when data were censored. OS and DSS comparisons were depicted as Kaplan-Meier and cumulative incidence plots, respectively. Multivariable Cox proportional hazards modeling and competing risk models, which accounted for clustering given the matched nature of the data, were used to compare OS and DSS in the TMT and RC groups, respectively.

\section{RESULTS}

\section{MDBCC Patient Demographics and Impact}

Characteristics of the 248 patients referred to the MDBCC from 2008 to 2012 are listed in Table 1; most patients were referred by urologists for a second opinion or for consideration of bladder preservation with TMT for MIBC. After MDBCC assessment, treatment recommendations were immediately possible for 51 patients (21\%). The remaining 197 patients (79\%) required

Table 1. Characteristics of Patients Assessed in the MDBCC From 2008 to 2012

\begin{tabular}{lc}
\hline \multicolumn{1}{c}{ Characteristic } & $\begin{array}{c}\text { No. }(\%) \text { of Patients } \\
(\mathrm{N}=248)\end{array}$ \\
\hline Sex & $192(77)$ \\
Male & $56(33)$ \\
Female & $71(37-95)$ \\
Median age, years (range) & \\
Stage at presentation & $78(32)$ \\
NMI (Ta, T1, Tis) & $162(65)$ \\
MI (T2, T3, T3) & $5(2)$ \\
Tx & $3(1)$ \\
PCA & $203(82)$ \\
Referring specialty & $21(8)$ \\
Urology & $10(4)$ \\
Family medicine & $7(3)$ \\
Medical oncology & $7(3)$ \\
Radiation oncology & \\
Other & $165(67)$ \\
Reason for referral & $15(6)$ \\
Second opinion & $15(6)$ \\
Unusualy histology & $50(20)$ \\
Radical cystectomy & $3(1)$ \\
TMT chemoradiation & \\
Other & \\
\hline
\end{tabular}

Abbreviations: MDBCC, multidisciplinary bladder cancer clinic; $\mathrm{MI}$, muscle invasive; NMI, non-muscle invasive; PCA, prostate cancer; TMT, trimodal therapy; Tx, stage undetermined.

*Patient data considered for subsequent propensity score comparison. additional investigation to recommend a final treatment plan. As a result of these additional measures $89(36 \%)$ of the 197 patients had a change in tumor stage (Fig 1) - 20 (44\%) of 45 patients from repeat TURBT, 47 (34\%) of 137 patients from additional imaging, and $22(17 \%)$ of 133 patients as a result of pathology review. Many patients had more than one investigation that contributed to stage change.

Eight $(16 \%)$ of the 51 patients for whom additional investigations were not recommended received a change in treatment plan based on multidisciplinary consultation and expert opinion. Likewise, $19(10 \%)$ of the 197 patients whose disease warranted additional investigations were advised about alternative treatment despite a lack of stage change on repeat investigation. Overall, additional investigations provided data that altered treatment recommendations 58 times, which resulted in treatment changes for 56 patients $(23 \%)$. A total of 83 patients $33 \%$ of all MDBCC attendees) were given different treatment plans after multidisciplinary consultation (56 patients who were reinvestigated with stage change, 19 patients who were reinvestigated without stage change, and eight patients without reinvestigation).

The final treatments provided were stratified as follows: RC was performed on 74 patients $(30 \%)$; 80 patients $(32 \%)$ were treated with radiotherapy, with or without chemotherapy; thirtyfive patients (14\%) were treated with intravesical therapy; 21 patients (9\%) received cystoscopic surveillance; 26 (10\%) received palliation. Other treatments were provided to 12 patients (5\%). Of the 80 patients who underwent radiotherapy, 24 (30\%) were deemed unfit for cystectomy on the basis of a comorbid status, whereas $56(70 \%)$ were candidates for cystectomy but chose bladder preservation.

\section{Comparison Between TMT and RC: Propensity Matched Analysis}

Of the 162 patients with MIBC, 49 of the 80 who received radiation-based therapy received full TMT (ie, maximal TURBT plus external-beam radiotherapy plus concomitant chemotherapy). The remaining 31 patients were either ineligible for cisplatin chemotherapy (ie, received radiation monotherapy), did not receive maximal TURBT, or had a disease with nonurothelial histology. To expand the cohort, 10 consecutive patients who received TMT from 2013 were added, which provided a total of 59 patients. Fifty-six patients had data that could be propensity matched to our institutional cystectomy database (2008 to 2013) with excellent matching characteristics and balance (Table 2). Appendix Figure A1 (online only) shows propensity scores after patients were matched, which demonstrates similarities between study groups. Rates of perioperative chemotherapy were well balanced with $37.5 \%$ (19.6\% neoadjuvant and $17.9 \%$ adjuvant) and $35.7 \%$ (all neoadjuvant) in the RC and TMT groups, respectively.

The median OS was 6.61 years, and there were no differences in OS $(P=.63)$ or DSS $(P=.49)$ on univariable analysis (Fig 2). Multivariable analysis that adjusted for $\mathrm{cT}$ and $\mathrm{cN}$ staging, presence of hydronephrosis, CIS, age, sex, ECOG status, and Charlson comorbidity score did not reveal significant differences in OS (TMT: hazard ratio $[\mathrm{HR}], 0.85 ; 95 \% \mathrm{CI}, 0.43$ to $1.66 ; P=.63$ ) or DSS (TMT: HR, $0.92 ; 95 \% \mathrm{CI}, 0.41$ to $2.04 ; P=.83$; data not shown). The 5-year expected DSS rates for RC and TMT were 


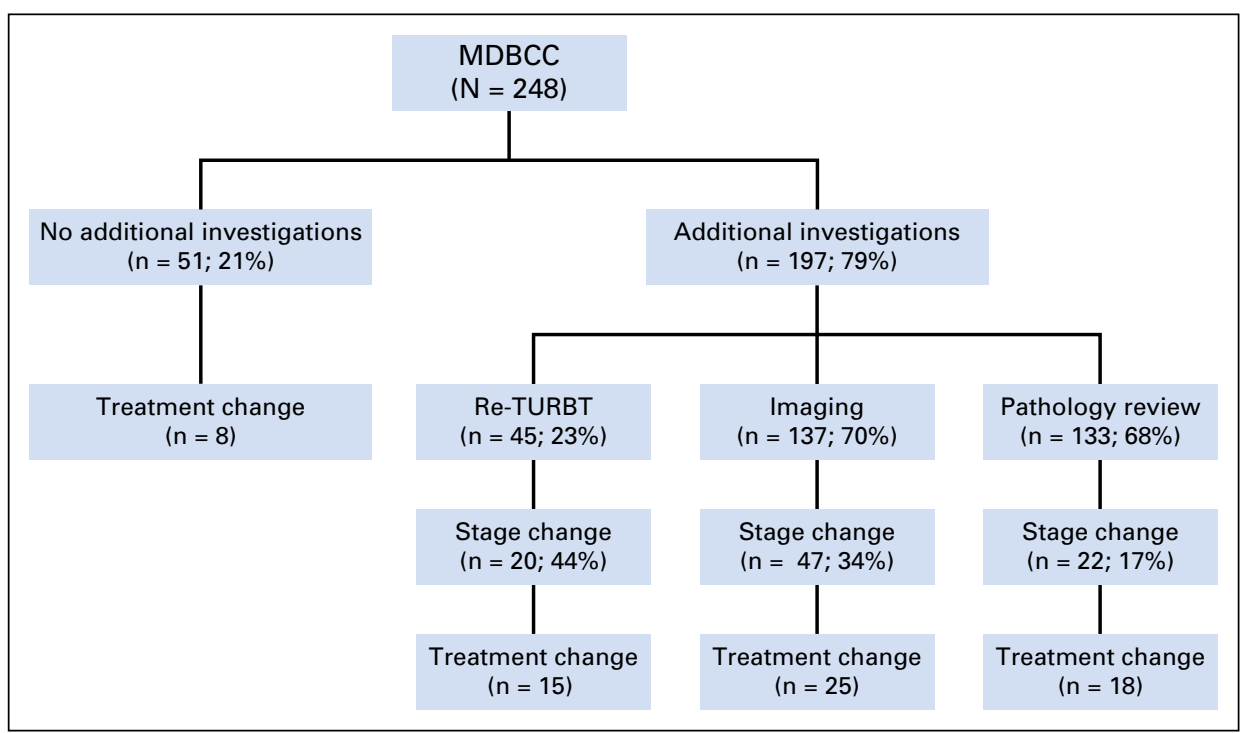

Fig 1. Changes in care and treatment plan after multidisciplinary bladder cancer clinic assessment of patients seen between 2008 and 2012. MDBCC, multidisciplinary bladder cancer clinic; Re-TURBT, repeat transurethral resection of bladder tumor.

$73.2 \%$ and $76.6 \%$, respectively $(P=.49)$. At a median follow-up of 4.51 years, there were 20 deaths $(35.7 \% ; 13$ as a result of $\mathrm{BC})$ in the RC group and 22 deaths $(39.3 \% ; 13$ as a result of BC) in the TMT group $(P=.84)$.

The overall recurrence rates in the $\mathrm{RC}$ and TMT groups were $38 \%$ and $59 \%$, respectively. Isolated intravesical recurrence

Table 2. Propensity Matched Characteristics of Patients With MIBC Undergoing Radical Cystectomy Versus Trimodal Therapy Chemoradiation

\begin{tabular}{|c|c|c|}
\hline \multirow[b]{2}{*}{ Characteristic } & \multicolumn{2}{|c|}{ No. (\%) of Patients } \\
\hline & $\begin{array}{l}\text { Cystectomy } \\
(n=56)\end{array}$ & $\begin{array}{c}\text { Chemoradiation } \\
(n=56)\end{array}$ \\
\hline \multicolumn{3}{|l|}{ Age at diagnosis, years } \\
\hline$\leq 60$ & $14(25)$ & $12(21.4)$ \\
\hline$>60$ & $42(75)$ & $44(78.6)$ \\
\hline \multicolumn{3}{|l|}{ Sex } \\
\hline Male & $41(73.2)$ & $40(71.4)$ \\
\hline Female & $15(26.8)$ & $16(28.6)$ \\
\hline \multicolumn{3}{|l|}{ T stage } \\
\hline Organ confined $(\mathrm{c} T \leq 2)$ & $41(73.2)$ & 38 (67.9) \\
\hline Not organ confined (cT > 2) & $15(26.8)$ & $18(32.1)$ \\
\hline \multicolumn{3}{|l|}{$\mathrm{N}$ stage } \\
\hline $\mathrm{cNO} / \mathrm{Nx}$ & $46(82.1)$ & $46(82.1)$ \\
\hline $\mathrm{cN}+(\mathrm{cN} 1, \mathrm{cN} 2, \mathrm{cN} 3)$ & $10(17.9)$ & $10(17.9)$ \\
\hline \multicolumn{3}{|l|}{$\mathrm{CIS}$} \\
\hline Absent & 38 (67.9) & 39 (69.6) \\
\hline Present & $18(32.1)$ & $17(30.4)$ \\
\hline \multicolumn{3}{|l|}{ Hydronephrosis } \\
\hline Absent & $49(87.5)$ & $48(85.7)$ \\
\hline Present & 7 (12.5) & 8 (14.3) \\
\hline \multicolumn{3}{|l|}{ ECOG performance status } \\
\hline 0 & $40(71.4)$ & $40(71.4)$ \\
\hline$\geq 1$ & $16(28.6)$ & $16(28.6)$ \\
\hline \multicolumn{3}{|l|}{ Charlson comorbidity score } \\
\hline$\leq 4$ & 39 (69.6) & $37(66.1)$ \\
\hline$>4$ & $17(30.4)$ & 19 (33.9) \\
\hline \multicolumn{3}{|l|}{ Treatment date } \\
\hline$\leq 2010$ & $30(53.6)$ & $24(42.9)$ \\
\hline$>2010$ & $26(46.4)$ & $32(57.1)$ \\
\hline
\end{tabular}

Abbreviations: CIS, carcinoma in situ; ECOG, Eastern Cooperative Oncology Group; MIBC, muscle-invasive bladder cancer. occurred in $17(30.3 \%)$ of patients who received TMT, with 12 $(21.4 \%)$ treated by endoscopic resection with or without intravesical bacillus Calmette-Guérin therapy. The salvage cystectomy rate for the TMT group was $10.7 \%$, and no cystectomy was required for bladder toxicity.

\section{DISCUSSION}

No randomized trials have directly compared RC and bladder preservation approaches with TMT, so there is a lack of level-1 evidence to guide patient management. The recent United Kingdom phase III trial, Selective Bladder Preservation Against Radical Excision (SPARE), unfortunately failed to accrue patients and resulted in premature closure. ${ }^{23}$ Most series of bladder preservation, with few exceptions, ${ }^{12,13}$ have included a limited number of cases per center, which compounds the lack of randomized data. $^{10,24}$

To overcome the limitations of published noncomparative retrospective studies, this study used propensity score matching with patients who underwent contemporary RC to generate comparable groups using stringent criteria. The data showed that TMT provides midterm survival outcomes comparable to $\mathrm{RC}$ in selected BC cases. This analysis supports the concept that multidisciplinary consultation with experts in $\mathrm{BC}$ care leads to improvements in $\mathrm{BC}$ staging, which may optimize treatment recommendations. Indeed, the MDBCC assessment resulted in treatment changes for $33 \%$ of referred patients, but, in the absence of a true control group, better outcomes remain unproven. Incorrect clinical staging, especially understaging, has been identified as a serious problem in BC, and improvements in the staging process are needed to achieve more accuracy and improved care for patients with BC. ${ }^{5,25}$

MDC enables assessment by multiple disciplines, with subsequent provision of specialty-specific input, enhances the likelihood of delivering optimal curative therapy ${ }^{26}$, adds an additional layer of checks and balances in the decision-making process to minimize errors in judgment, and maximizes the probability of 
A

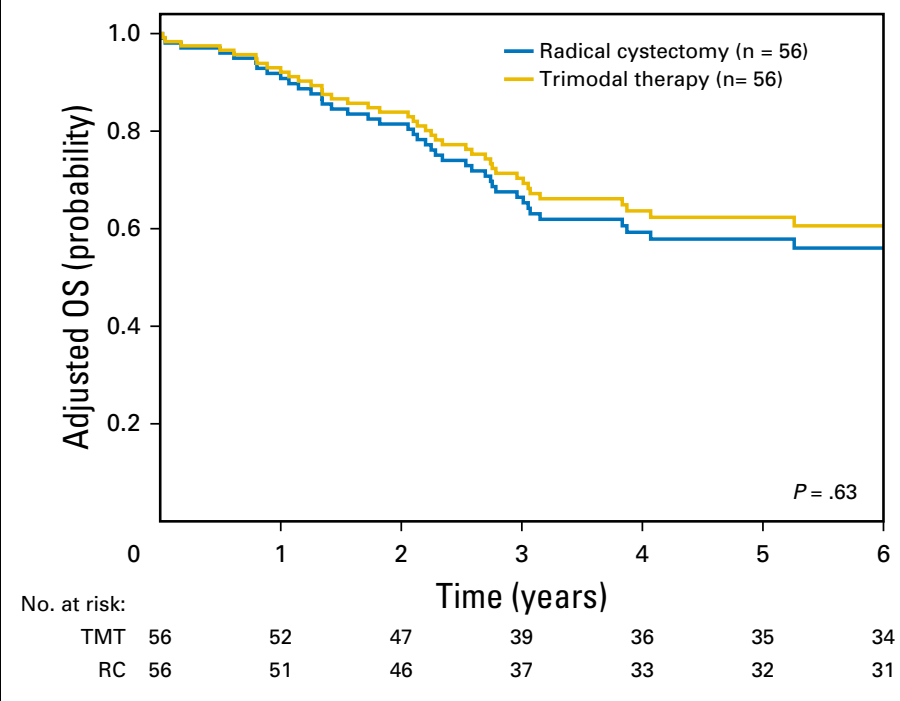

B

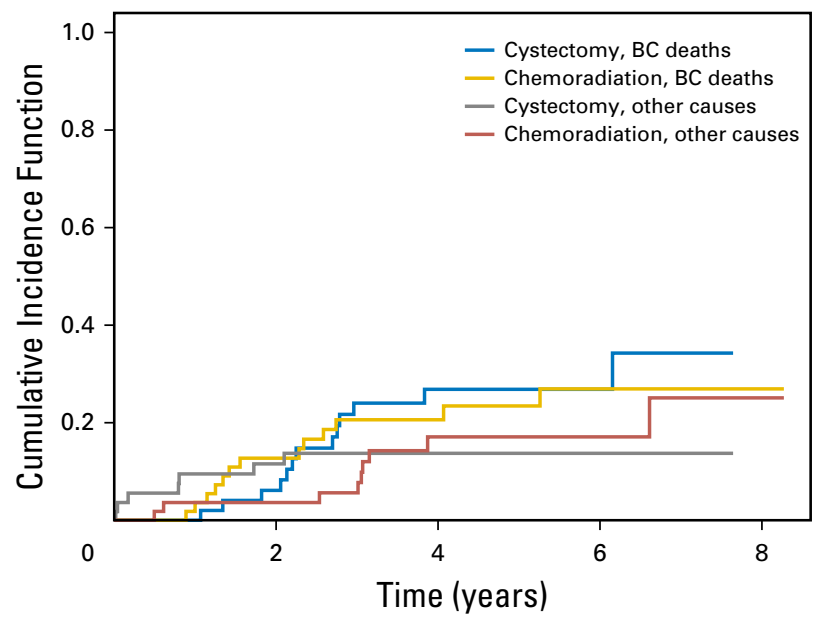

Fig 2. Propensity score matched survival outcomes of patients after radical cystectomy versus trimodality therapy chemoradiation. (A) Kaplan-Meier curve of overall

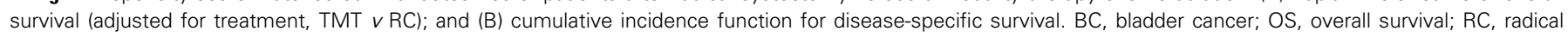
cystectomy; TMT, trimodal therapy.

adopting treatment strategies that are based on the highest levels of evidence. ${ }^{27}$ Data from oncologic literature in other cancer types ${ }^{6,8,9}$ support improved survival outcomes in patients who receive care via multidisciplinary team approaches, and this may also be the case for patients with BC.

Despite the purported benefits of MDC in oncology, its uptake in $\mathrm{BC}$ has been slow and may be responsible for some degree of undertreatment of many patients with MIBC. For example, a recent observational study that used propensity score analysis to compare the effectiveness of AC versus observation post cystectomy in 5,653 patients with pathologic T3-4 and/or pathologic node-positive $\mathrm{BC}$ demonstrated that a mere $23 \%$ received AC. AC was associated with improved survival in patients with locally advanced BC (HR, 0.70; 95\% CI, 0.64 to 0.76$).{ }^{28}$ Similar underuse of NAC in patients with MIBC has been reported. ${ }^{26,27}$ Data from the SEER-Medicare database on MIBC treatment show that a disturbing $51 \%$ of patients did not receive any definitive therapy. ${ }^{28}$ Many of these patients may have been eligible for some form of multimodal therapy, although they may have been ineligible for RC.

Critics of TMT point to its potential lack of efficacy compared with RC or the absence of long-term data. A pooled analysis of six historical Radiation Therapy Oncology Group single-arm studies demonstrated a similar 5-year DSS rate of 71\% compared with modern MIBC cystectomy series. ${ }^{10}$ However, cystectomy series are not limited to select patients with smaller, unifocal tumors without CIS or hydronephrosis in which 5-year DSS rates with surgery may be closer to $80 \%,{ }^{29,30}$ so robust or definitive conclusions are limited. Furthermore, although contemporary TMT series involve more highly selected populations, historical RT series have included a large proportion of patients not suitable for RC because of age, comorbidities, and/or disease extent, which could explain lower-than-expected survival rates in these series; this difference further clouds potential comparisons.

In this study, the near identical 5-year DSS for RC and TMT are in keeping with modern MIBC outcomes reported in cystectomy series $^{31,32}$ and demonstrate excellent and comparable results of modern TMT in selected patients with MIBC. The series had a $10.7 \%$ salvage cystectomy rate for high-risk NMIBC and MIBC recurrences after TMT. The lack of toxicity-related cystectomies supports the tolerability of the regimen. The success of TMT requires the expertise not only of radiation and medical oncologists to deliver treatment but also of urologic oncologists experienced in surveying postradiation bladders (in which differentiation between posttreatment radiation changes and tumor recurrence is not always straightforward) and who can safely perform salvage cystectomy.

Limitations of this study include its retrospective nature and the associated selection bias, which confounds by indication. To overcome this, propensity-matching techniques were applied to create comparable TMT and RC cohorts. Even with this methodology, unknown confounders may not be accounted for. Nevertheless, balanced, matched comparisons were created on the basis of clinical factors (cT and $\mathrm{cN}$ stage, age, comorbidity, hydronephrosis, and CIS) that are widely reported to influence outcome (Appendix Fig A1). Second, the number of patients included in the propensity score analysis was limited. The study included only 112 patients, so larger cohorts are required to validate the results, because limited statistical power may have contributed to the statistically nonsignificant comparisons. The DSS and OS rates were nearly identical between surgery and chemoradiation at 5 years (DSS, 73\% $v 77 \%$, respectively), so it seems rather unlikely that expansion of the cohort size would dramatically modify these initial findings. Third, the median 
follow-up of 4.51 years may be considered limited, although, in patients with $\mathrm{BC}$, pertinent clinical outcomes are often reached with this length of follow-up. Fourth, we had no quality-of-life data but have initiated the collection of these data. Fifth, this is a singleinstitution study, and there is the possibility that different centers may experience different outcomes. Sixth, because the study lacked a true control arm of non-MDBCC care we cannot definitively prove that changes we implemented in care as a result of multidisciplinary consultation resulted in improved care. Change in stage was used as a surrogate for appropriate treatment delivery. Finally, the best method by which MDC should be provided cannot be determined from this report. In the MDBCC, assessment and consultation occur in parallel, with multiple specialists providing input simultaneously, although BC medical oncologists and expert GU pathologists were not physically present in the clinic at the same time. Lack of a full multidisciplinary complement in the same physical space did not affect their input. Specifically, we have increased use of NAC from $7.7 \%$ to $47.6 \%$ with MDBCC initiation, ${ }^{27}$ and well over half of the patients undergo pathologic specimen review, which suggests that this limitation does not preclude MDC. In our clinic, cystoscopic assessment of patients treated with TMT by urologic and radiation oncologists present simultaneously enables joint decision making regarding the need for additional investigations and/or procedures. Whether this model provides improved outcomes compared with consultations done sequentially is unclear. Optimal outcomes, we believe, can be achieved through multidisciplinary collaboration by GU-trained oncology caregivers, regardless of how care is provided.

In conclusion, the advantage of MDC is the ability to review all treatment options with patients in an unbiased fashion. We observed a change in tumor staging in more than a third of patients after review in the MDBCC with subsequent modifications of the initial treatment plans. These results show that selected patients who undergo TMT experience outcomes similar to those who undergo RC. Patients eligible for TMT should be offered the opportunity to discuss all therapeutic alternatives before treatment is selected. Rather than pitting surgery against TMT, choice of the right therapeutic option for the right patient with $\mathrm{BC}$ in the setting of MDC should be encouraged.

\section{AUTHORS' DISCLOSURES OF POTENTIAL CONFLICTS} OF INTEREST

Disclosures provided by the authors are available with this article at jco.org.

\section{AUTHOR CONTRIBUTIONS}

Conception and design: Girish S. Kulkarni, Thomas Hermanns, Peter Chung, Robert G. Bristow, Michael Milosevic, Padraig Warde, Neil E. Fleshner, Michael A.S. Jewett, Alexandre R. Zlotta

Collection and assembly of data: Thomas Hermanns, Yanliang Wei, Bimal Bhindi, Paul Athanasopoulos, Peter J. Bostrom, Cynthia Kuk, Kathy Li, Theodorus H. van der Kwast

Data analysis and interpretation: Thomas Hermanns, Yanliang Wei, Raj Satkunasivam, Peter J. Bostrom, Arnoud J. Templeton, Srikala S. Sridhar, Robert G. Bristow, Shaheena Bashir, Alexandre R. Zlotta

Manuscript writing: All authors

Final approval of manuscript: All authors

Accountable for all aspects of the work: All authors

\section{REFERENCES}

1. Kulkarni GS, Urbach DR, Austin PC, et al: Higher surgeon and hospital volume improves longterm survival after radical cystectomy. Cancer 119 : 3546-3554, 2013

2. Christodouleas JP, Baumann BC, He J, et al: Optimizing bladder cancer locoregional failure risk stratification after radical cystectomy using SWOG 8710. Cancer 120:1272-1280, 2014

3. Hermans TJ, Fransen van de Putte EE, Horenblas $S$, et al: Perioperative treatment and radical cystectomy for bladder cancer: A population-based trend analysis of 10,338 patients in the Netherlands. Eur J Cancer 54:18-26, 2016

4. Grossman HB, Natale RB, Tangen CM, et al: Neoadjuvant chemotherapy plus cystectomy compared with cystectomy alone for locally advanced bladder cancer. N Engl J Med 349:859-866, 2003

5. Turker $\mathrm{P}$, Bostrom $\mathrm{PJ}$, Wroclawski $\mathrm{ML}$, et al: Upstaging of urothelial cancer at the time of radical cystectomy: Factors associated with upstaging and its effect on outcome. BJU Int 110:804-811, 2012

6. Prades J, Remue E, van Hoof E, et al: Is it worth reorganising cancer services on the basis of multidisciplinary teams (MDTs)? A systematic review of the objectives and organisation of MDTs and their impact on patient outcomes. Health Policy 119: 464-474, 2015

7. Pillay $B$, Wootten $A C$, Crowe $H$, et al: The impact of multidisciplinary team meetings on patient assessment, management and outcomes in oncology settings: A systematic review of the literature. Cancer Treat Rev 42:56-72, 2016

8. De Palma R, Liberati $A$, Ciccone G, et al: Developing clinical recommendations for breast, colorectal, and lung cancer adjuvant treatments using the GRADE system: A study from the Programma Ricerca e Innovazione Emilia Romagna oncology research group. J Clin Oncol 26:1033-1039, 2008

9. Berho M, Narang R, Van Koughnett JA, et al: Modern multidisciplinary perioperative management of rectal cancer. JAMA Surg 150:260-266, 2015

10. Mak RH, Hunt $D$, Shipley WU, et al: Long-term outcomes in patients with muscle-invasive bladder cancer after selective bladder-preserving combinedmodality therapy: A pooled analysis of Radiation Therapy Oncology Group protocols 8802, 8903, 9506, 9706, 9906, and 0233. J Clin Oncol 32: 3801-3809, 2014

11. Chung PW, Bristow RG, Milosevic MF, et al: Long-term outcome of radiation-based conservation therapy for invasive bladder cancer. Urol Oncol 25: 303-309, 2007

12. Efstathiou JA, Spiegel DY, Shipley WU, et al: Long-term outcomes of selective bladder preservation by combined-modality therapy for invasive bladder cancer: The MGH experience. Eur Urol 61: 705-711, 2012

13. James ND, Hussain SA, Hall E, et al: Radiotherapy with or without chemotherapy in muscleinvasive bladder cancer. N Engl J Med 366: 1477-1488, 2012

14. Gofrit $O N$, Nof $R$, Meirovitz $A$, et al: Radical cystectomy vs. chemoradiation in T2-4aNOMO bladder cancer: A case-control study. Urol Oncol 33: 19.e1-19.e5, 2015

15. Bostrom $\mathrm{PJ}$, Toren $\mathrm{PJ}, \mathrm{XiH}_{\mathrm{H}}$, et al: Point-of-care clinical documentation: assessment of a bladder cancer informatics tool (eCancerCareBladder): A randomized controlled study of efficacy, efficiency and user friendliness compared with standard electronic medical records. J Am Med Inform Assoc 18: 835-841, 2011

16. Bhindi B, Yu J, Kuk C, et al: The importance of surgeon characteristics on impacting oncologic outcomes for patients undergoing radical cystectomy. J Urol 192:714-719, 2014

17. Galsky MD, Hahn NM, Rosenberg J, et al: Treatment of patients with metastatic urothelial cancer unfit for cisplatin-based chemotherapy. J Clin Oncol 29:2432-2438, 2011

18. Lamm DL, Blumenstein BA, Crissman JD et al: Maintenance bacillus Calmette-Guerin immunotherapy for recurrent TA, T1, and carcinoma in situ transitional cell carcinoma of the bladder: A randomized Southwest Oncology Group study. J Urol 163:1124-1129, 2000

19. Kassouf W, Kamat AM, Zlotta A, et al: Canadian guidelines for treatment of non-muscle invasive bladder cancer: A focus on intravesical therapy. Can Urol Assoc J 4:168-173, 2010

20. Hall MC, Chang SS, Dalbagni G, et al: Guideline for the management of nonmuscle invasive bladder cancer (stages Ta, T1, and Tis): 2007 update. J Urol 178:2314-2330, 2007

21. Babjuk $M$, Burger $M$, Zigeuner $R$, et al: EAU guidelines on non-muscle-invasive urothelial 
carcinoma of the bladder: Update 2013. Eur Urol 64: 639-653, 2013

22. Heinze $G$, Jüni $P$ : An overview of the objectives of and the approaches to propensity score analyses. Eur Heart J 32:1704-1708, 2011

23. Huddart RA, Hall E, Lewis R, et al: Life and death of spare (selective bladder preservation against radical excision): Reflections on why the spare trial closed. BJU Int 106:753-755, 2010

24. Choudhury $A$, Swindell $R$, Logue JP, et al: Phase II study of conformal hypofractionated radiotherapy with concurrent gemcitabine in muscleinvasive bladder cancer. J Clin Oncol 29:733-738, 2011

25. Bostrom PJ, van Rhijn BWG, Fleshner $N$, et al: Staging and staging errors in bladder cancer. Eur Urol Suppl 9:2-9, 2010
26. Booth CM, Siemens DR, Peng $Y$, et al: Patterns of referral for perioperative chemotherapy among patients with muscle-invasive bladder cancer: A population-based study. Urol Oncol 32:1200-1208, 2014

27. Nayan $M$, Bhindi $B, Y u$ JL, et al: The initiation of a multidisciplinary bladder cancer clinic and the uptake of neoadjuvant chemotherapy: A time-series analysis. Can Urol Assoc J 10:25-30, 2016

28. Gore JL, Litwin MS, Lai J, et al: Use of radical cystectomy for patients with invasive bladder cancer. J Natl Cancer Inst 102:802-811, 2010

29. Tilki D, Reich O, Karakiewicz Pl, et al: Validation of the AJCC TNM substaging of pT2 bladder cancer: Deep muscle invasion is associated with significantly worse outcome. Eur Urol 58:112-117, 2010
30. Gakis G, Schilling D, Renninger $M$, et al: Comparison of the new American Joint Committee on Cancer substratification in node-negative pT2 urothelial carcinoma of the bladder: Analysis of patient outcomes in a contemporary series. BJU Int 107:919-923, 2011

31. Zehnder P, Studer UE, Skinner EC, et al: Super extended versus extended pelvic lymph node dissection in patients undergoing radical cystectomy for bladder cancer: A comparative study. J Urol 186: 1261-1268, 2011

32. Sonpavde G, Khan MM, Lerner SP, et al: Disease-free survival at 2 or 3 years correlates with 5 year overall survival of patients undergoing radical cystectomy for muscle invasive bladder cancer. J Urol 185:456-461, 2011

\section{Affiliations}

Girish S. Kulkarni, Thomas Hermanns, Yanliang Wei, Bimal Bhindi, Raj Satkunasivam, Paul Athanasopoulos, Peter J. Bostrom, Kathy Li, Arnoud J. Templeton, Srikala S. Sridhar, Peter Chung, Robert G. Bristow, Michael Milosevic, Padraig Warde, Neil E. Fleshner, Michael A.S. Jewett, Shaheena Bashir, and Alexandre R. Zlotta, Princess Margaret Cancer Center, University Health Network, University of Toronto; Cynthia Kuk and Alexandre R. Zlotta, Mount Sinai Hospital, University of Toronto; Theodorus H. Van Der Kwast, University Health Network, University of Toronto, Toronto, ON, Canada.

\section{Prior Presentation}

Presented in part at the 2016 Genitourinary Cancers Symposium, San Francisco, CA, January 7-9, 2016.

\section{ASCO's Quality Assessment Tool for Community Research Programs}

As part of ASCO's mission to ensure the highest quality of cancer care and research, the Research Community Forum has developed the ASCO Research Program Quality Assessment Tool. Access the tool today to learn about key components of an internal quality assessment program, conduct a quality assessment of your site, and use the checklist tool, templates, references, and resources.

Visit asco.org/researchcommunityforum for more information. 
Propensity Score Analysis of Radical Cystectomy Versus Bladder-Sparing Trimodal Therapy in the Setting of a Multidisciplinary Bladder Cancer Clinic

The following represents disclosure information provided by authors of this manuscript. All relationships are considered compensated. Relationships are self-held unless noted. I = Immediate Family Member, Inst = My Institution. Relationships may not relate to the subject matter of this manuscript. For more information about ASCO's conflict of interest policy, please refer to www.asco.org/rwc or ascopubs.org/jco/site/ifc.

Girish S. Kulkarni

No relationship to disclose

\section{Thomas Hermanns}

No relationship to disclose

Yanliang Wei

No relationship to disclose

Bimal Bhindi

No relationship to disclose

Raj Satkunasivam

No relationship to disclose

Paul Athanasopoulos

No relationship to disclose

\section{Peter J. Bostrom}

No relationship to disclose

\section{Cynthia Kuk}

No relationship to disclose

Kathy Li

No relationship to disclose

Arnoud J. Templeton

Consulting or Advisory Role: Bristol-Myers Squibb (Inst)

Srikala S. Sridhar

Consulting or Advisory Role: Roche, Astra Zeneca, Merck, Bayer, Pfizer

Theodorus H. van der Kwast

No relationship to disclose

Peter Chung

Honoraria: Abbvie

Research Funding: Sanofi Canada

\section{Robert G. Bristow \\ Research Funding: GenomeDx}

Michael Milosevic

No relationship to disclose

Padraig Warde

No relationship to disclose

Neil E. Fleshner

Honoraria: Amgen, Janssen, Bayer, Sanofi, Abbvie, Ferring, Astellas, Medivation, Hybridype Imaging Technologies

Research Funding: Ferring (Inst), Astellas (Inst), Janssen (Inst), Amgen (Inst), Bavarian Nordic (Inst), Bayer (Inst), Medivation (Inst), Nucleix (Inst), Progenics (Inst), Sanofi (Inst), Spectracure AB (Inst)

\section{Michael A.S. Jewett}

Employment: Impact Genetics (I)

Leadership: Impact Genetics (I)

Stock or Other Ownership: Theralase Technologies

Honoraria: Pfizer, Myriad Genetics

Consulting or Advisory Role: Theralase Technologies

Research Funding: Olympus Medical Systems (Inst)

Patents, Royalties, Other Intellectual Property: Patent held for a new radiofrequency ablation device

\section{Shaheena Bashir}

No relationship to disclose

\section{Alexandre R. Zlotta}

Stock or Other Ownership: iTP Biomedica

Consulting or Advisory Role: Ferring, BioSyent, Sanofi

Research Funding: Ferring (Inst), Sanofi (Inst)

Patents, Royalties, Other Intellectual Property: iTP Biomedica (Inst) 


\section{Acknowledgment}

We thank Annette Erlich for her help with the administrative and publishing aspects of this manuscript.

\section{Appendix}

\section{Methods}

Technical statistical appendix. A total of 59 patients who underwent trimodal therapy (TMT) for bladder cancer (BC) in a multidisciplinary bladder cancer clinic (MDBCC) from 2008 to 2013 were identified. These patients were matched to patients who underwent radical cystectomy $(\mathrm{RC})$ during the same time frame, via propensity scores. Specifically, a logistic regression propensity score model of the type of treatment modality received (TMT $v$ RC) was created, and the following variables were included in the model: age, sex, cT and cN stage (clinical TNM stage), presence of carcinoma in situ (CIS), presence of hydronephrosis, Eastern Cooperative Onoclogy Group (ECOG) status, Charlson comorbidity score, and treatment date. Matching across the two groups was achieved with a 0.2-width caliper of the standard deviation of the logit of the propensity scores to reduce bias because of confounding. The propensity score distributions for the groups both before and after matching are shown in Appendix Figure A1; Figure A1A demonstrates an initial dissimilarity across the two groups on the basis of propensity score distributions. However, after matching, the distributions of the propensity scores for the two groups (Appendix Fig A1B) were quite homogeneous and demonstrated the adequacy of the propensity score model to achieve balance. Table 2 also depicts the similarity across groups after propensity score matching.

To compare overall survival in both groups, a multivariable Cox proportional hazards model was created to estimate the hazard of death as a result of $\mathrm{BC}$ after adjustment for other risk factors. The same variables used to generate the propensity score (ie, age, sex, $\mathrm{cT}$ and $\mathrm{cN}$ stage [clinical TNM stage], presence of CIS, presence of hydronephrosis, ECOG status, Charlson comorbidity score, and treatment date) were included as covariates in the model. Because of the paired nature of the 1:1 matched data, matched identify of the pairs as a cluster variable was used. This gave robust estimates of variance for the correlated matched data.

In the case of the competing risk regression for disease-specific survival, correlated matched pairs were accounted for by using competing risk regression for clustered data. This method extends the proportional hazards model of Fine and Gray for subdistribution to accommodate situations in which the failure times within a cluster might be correlated, because the study subjects from the same cluster share common factors (Fine JP, Gray RJ: J Am Stat Assoc 94:496-509, 1999). This model directly assesses the effect of covariates on the subdistribution of a particular type of failure in a competing risk setting. Again, covariates included in the competing risk model were age, sex, $\mathrm{cT}$ and $\mathrm{cN}$ stage (clinical TNM stage), presence of CIS, presence of hydronephrosis, ECOG status, Charlson comorbidity score, and treatment date.
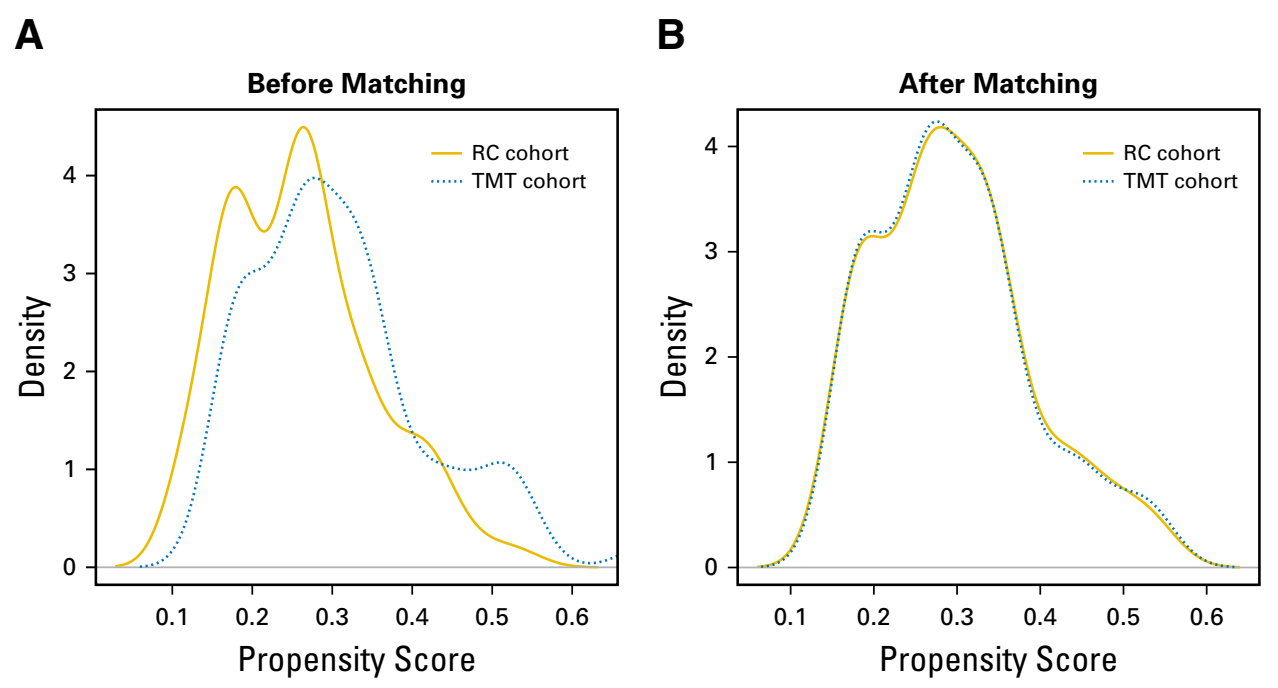

Fig A1. Propensity score distributions (A) before matching, and (B) after matching. RC, radical cystectomy; TMT, trimodal therapy. 